\title{
The structure of line-driven winds
}

\author{
L. B. Lucy \\ Astrophysics Group, Blackett Laboratory, Imperial College London, Prince Consort Road, London SW7 2AZ, UK
}

e-mail: 1.lucy@imperial.ac.uk

Received 6 July 2007 / Accepted 11 September 2007

\section{ABSTRACT}

\begin{abstract}
Following procedures pioneered by Castor et al. (1975, ApJ, 195, 157, [CAK]), spherically-symmetric supersonic winds for O stars are computed for matching to plane-parallel moving reversing layers (RL's) from Paper I (Lucy 2007, A\&A, 468, 649). In contrast to a CAK wind, each of these solutions is singularity-free, thus allowing its mass-loss rate to be fixed by the regularity condition at the sonic point within the RL. Moreover, information propagation in these winds by radiative-acoustic waves is everywhere outwardlydirected, justifying the implicit assumption in Paper I that transonic flows are unaffected by inwardly-directed wave motions.
\end{abstract}

Key words. stars: early-type - stars: mass-loss - stars: winds, outflows

\section{Introduction}

In a recent paper (Lucy 2007, [Paper I]), models of moving reversing layers (RL's) for O stars were used to investigate the role of photospheric turbulence in regulating the mass flux $J$ that can be accelerated to supersonic velocities. According to the underlying theory, the integro- differental equations describing stationary plane-parallel outflow have $J$ as an eigenvalue, and this is determined by demanding regularity at the sonic point (Lucy \& Solomon 1970).

The solutions in Paper I were followed to velocities $v \sim 4 a$, where $a$ is the isothermal speed of sound. Left in abeyance, therefore, was the question of matching to solutions describing the highly supersonic, spherically-symmetric winds where $\mathrm{P}$ Cygni lines are formed.

Many investigators of line-driven winds follow Castor et al. (1975, [CAK]) in using the Sobolev approximation to compute $g_{\ell}$, the radiation force per gm due to lines. Since this approximation is valid at the highest velocites reached by the solutions in Paper I, continuation to $r=\infty$ with a CAK solution would seem to be appropriate. But matching a CAK wind to a moving-RL is in general impossible. Given a star's basic parameters, namely composition, mass $\mathcal{M}$, radius $R$ and luminosity $L$, the regularity condition at the CAK critical point admits only one solution, with mass-loss rate $\Phi_{\mathrm{CAK}}$ as its eigenvalue. Since the movingRL already specifies that $\Phi=\Phi_{\mathrm{RL}}=4 \pi R^{2} J$, we will in general find that $\Phi_{\mathrm{CAK}} \neq \Phi_{\mathrm{RL}}$.

This matching problem is the topic of this paper. At first sight, there are two approaches. If the moving-RL is the accepted starting point, then continuation to higher velocities requires solution of the basic integro-differential system since a CAK singularity then no longer exists (Lucy 1975) and so $\Phi$ is not constrained by a regularity condition at supersonic velocity. On the other hand, if the unique CAK solution is the accepted starting point, then some mechanism must be identified that can force a structural adjustment of the RL so that $J=\Phi_{\mathrm{CAK}} / 4 \pi R^{2}$.

\section{Supersonic winds}

This paper's basic premise is that the critical point that determines the eigenvalue $\Phi$ of a stationary line-driven wind is the sonic point and not the CAK critical point. In this section, therefore, a model of the spherically-symmetric supersonic zone is developed for matching to the plane-parallel sub- and transonic models of Paper I.

\subsection{The model}

Given that the aim is to resolve the paradox of two distict procedures for predicting $\Phi$, a fairly simple model is adopted: numerical precision is surely not needed to settle a question of principle. Accordingly, the supersonic wind is assumed to be isothermal with $T=0.75 T_{\text {eff }}$ and $\mathrm{H}$ and $\mathrm{He}$ are assumed to be full ionized.

With sphericity now included, the equation of continuity has the integral

$4 \pi r^{2} \rho v=\Phi$

and the equation of motion can then be written as

$\left(v^{2}-a^{2}\right) \frac{1}{v} \frac{\mathrm{d} v}{\mathrm{~d} r}=\frac{2 a^{2}}{r}+g_{\ell}-g_{*}$

where $g_{*}=\left(1-\Gamma_{\mathrm{e}}\right) g$.

\subsection{Radiation force}

In Sect. 1, a plausible argument was given that the calculation of $g_{\ell}$ must be based on non-Sobolev transfer since otherwise a CAK singularity will be encountered in the supersonic flow. Notwithstanding this argument, $g_{\ell}$ is now derived using the Sobolev approximation. The assumptions are as follows:

1) As in CAK, $v$ is assumed to be a monotonically increasing function of $r$, and multi-line scattering is neglected. Accordingly, each line interacts with dilute but unattenuated photospheric radiation. 
2) The CAK assumption of radial streaming for photospheric radiation is not adopted. Instead, the finite cone occupied by this radiation is accounted for using modified Eddington approximations (Lucy 1971). The importance of this departure from CAK was demonstrated by Pauldrach et al. (1986), but their procedure of introducing this effect via a correction factor $C F$ is not followed.

3) The CAK procedure of approximating the summed contributions of numerous lines to $g_{\ell}$ by means of an analytic force multiplier function $M(t)$ is not followed. This approximation has the merit of computational economy and, more importantly, allows a fairly straightforward analysis of solution domains and singular points. But this analysis becomes significantly more complicated when $C F$ is included, and yet further complicated when the $M(t)$ parameters vary with radius as in WM-basic models (Pauldrach et al. 2001). Accordingly, the force multiplier approach is dropped and replaced by direct summation.

The resulting force per gm due to line transitions is

$g_{\ell}=\sum_{i} g_{i}$

where $g_{i}$, the contribution from the $i$ th line, is given by (Lucy 1971)

$g_{i}=\frac{\kappa_{i}}{c} \pi F_{i} x^{2} \frac{1-\mathrm{e}^{-\tau_{i, 1}}}{\tau_{i, 1}}$.

Here $\pi F_{i}$ is the flux emitted at the line's rest frequency $v_{i}$ by the photosphere located at $x=R / r=1$. Line formation is accounted for through the factor containing the Sobolev optical depth given by

$\tau_{i, 1}=\frac{\kappa_{i} \rho c}{v_{i}} /\left[\mu_{1}^{2} \frac{\mathrm{d} v}{\mathrm{~d} r}+\left(1-\mu_{1}^{2}\right) \frac{v}{r}\right]$

and evaluated at direction cosine $\mu_{1}=\sqrt{1-x^{2} / 2}$. The properties of the transition $l \rightarrow u$ enter through $\kappa_{i}$, given in standard notation by

$\kappa_{i} \rho=\frac{\pi e^{2}}{m_{\mathrm{e}} c} n_{l} f_{l u}\left(1-\frac{g_{l} n_{u}}{g_{u} n_{l}}\right)$.

The above formula for $g_{i}$ corresponds to a 1-point quadrature formula for the integration over $\mu$ using the beam that leaves the photosphere with $\mu_{1}=1 / \sqrt{2}$ and assuming no limb-darkening.

As in Paper I, the data for the $\sim 10^{5}$ lines contributing to $g_{\ell}$ are from Kurucz \& Bell (1995). The fluxes $\pi F_{i}$ are derived from the emergent fluxes of TLUSTY models (Lanz \& Hubeny 2003) by smoothing with a box filter of width $1000 \mathrm{~km} \mathrm{~s}^{-1}$. Photospheric line-blanketing is therefore included but windblanketing is neglected.

The treatment of ionization and excitation is basically that of Abbott \& Lucy (1985) as updated in Lucy (1999), with dilution as the dominant NLTE effect. Chemical composition is as stated in Sect. 2.1 of Paper I.

\subsection{The CAK ansatz}

When derived as above, $g_{\ell}(r)$ depends only on the local properties of the wind at $r$, including the derivative $v^{\prime}=\mathrm{d} v / \mathrm{d} r$. But line formation is non-local, occurring over a finite velocity interval corresponding to a few times the mean thermal speed of the absorbing ions. Accordingly, $v^{\prime}$ in Eq. (5) - the Sobolev derivative
- represents an average over this interval. As such, it does not have the same standing as $v^{\prime}$ in Eq. (2) - the Newtonian derivative.

Though doubtless aware of this distinction, CAK nevertheless took the bold step of assigning equal status to these two derivatives. In consequence, the right-hand side of Eq. (2) is a function of $v^{\prime}$, which cannot then be extracted algebraically.

When making an approximation, even if apparently well justified, we should be concerned whenever the mathematical nature of the problem changes, resulting in the addition or removal of crucial aspects of the solutions. The Sobolev approximation is certainly plausible for high velocity outflows and has been widely and usefully employed for transfer problems in stellar winds and supernovae. But when incorporated into the dynamical theory of winds via the CAK ansatz, a system of integro-differental equations is replaced by an ordinary differential equation (ODE). This results in the following changes to the solutions: 1) the sonic point is no longer a critical point; and 2) a new (CAK) critical point appears at supersonic velocities. Since critical points are certainly crucial, these changes should alert us to the possibility that the CAK solution may not be a good approximation to the corresponding solution of the basic integro-differential system. Despite this reservation, the above modified CAK theory is used in Sect. 3 to investgate supersonic winds.

\subsection{Initial condition}

Given the CAK ansatz, Eq. (2) reduces to a non-linear first order ODE with dependent variable $v$. Since the Sobolev approximation is not well justified in the sub- and transonic flow, an initial condition $v=v_{1}$ at $x=x_{1}$ is imposed at a point $P_{1}$ where the bulk motion is already highly supersonic. This point is selected from the appropriate RL of Paper I.

By thus choosing a supersonic matching point, we also avoid CAK theory's elimination of the sonic point as a critical point. Its correct role appears here implicitly via the initial condition derived from the RL, where regularity at the critical point $v=a$ has already been imposed to determine $J$.

The mass-loss rate $\Phi$ is left as a free parameter except when set $=\Phi_{\mathrm{RL}}$, the rate predicted by the moving-RL.

\section{Solution topology}

In this section, the topology of the solutions of the nonlinear ODE defined in Sect. 2 is investigated. A general analytic discussion such as that of CAK is not possible because of the dropping of their simplifying assumptions. Instead, the topology is investigated numerically but only along and in the neighbourhood of the trajectories $v(x ; \Phi)$ for specific models.

Following CAK, we write Eq. (2) formally as

$Q\left(v^{\prime}, v, x ; \Phi\right)=0$.

This differential system has singular points on loci defined by

$\frac{\partial Q}{\partial v^{\prime}}=\frac{v^{2}-a^{2}}{v}-\frac{\partial g_{\ell}}{\partial v^{\prime}}=0$

Integration through such points is possible only if a regularity condition is satisfied.

For graphical presentation, it is convenient to make $Q$ and $v^{\prime}$ dimensionless by dividing by $g_{*}(r)$ and $v_{\text {esc }} / R$, respectively. In addition, since $v^{\prime}$ is constrained to be $>0$ by the assumption of a monotonic velocity law (Sect. 2.2), we define $z$ to be the logarithm of the dimensionless $v^{\prime}$. 


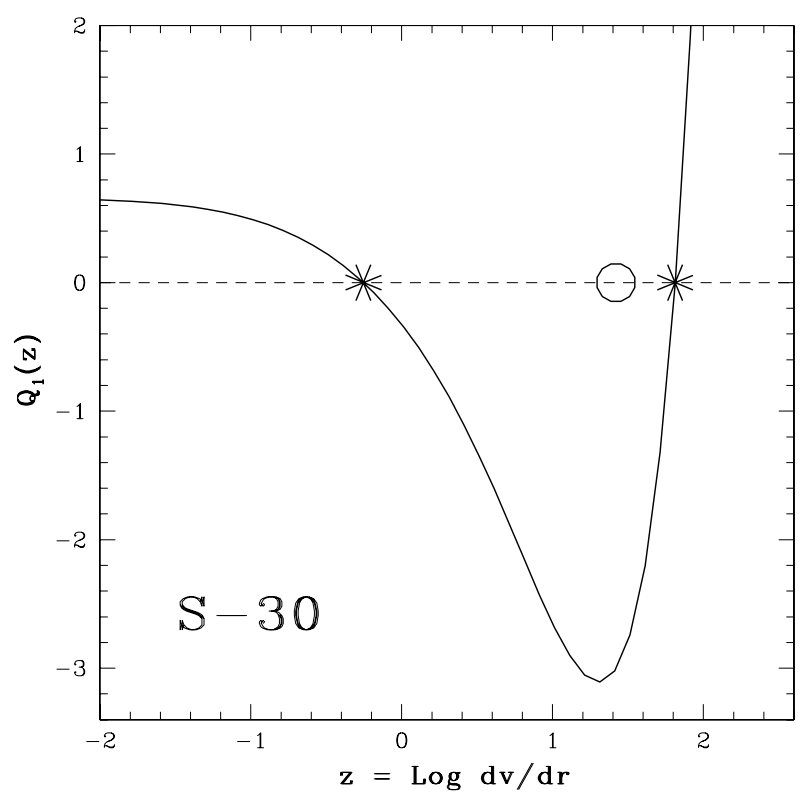

Fig. 1. Search for velocity gradient at $P_{1}$ for model S-30 when $\Phi=\Phi_{\mathrm{RL}}$. The asterisks indicate the two roots of the equation $Q_{1}=0$. The open circle is the prediction of the moving-RL from Paper I.

\subsection{A particular model}

Model S-30 of Pauldrach et al. (2001) is selected for detailed discussion. The parameters are $T_{\text {eff }}=30000 \mathrm{~K}, \log g=3$, and $R / R_{\odot}=27$, giving $v_{\mathrm{esc}}=\sqrt{2 g_{*} R}=423 \mathrm{~km} \mathrm{~s}^{-1}$.

According to Paper I, for microturbulent velocity $v_{\mathrm{t}}=$ $10 \mathrm{~km} \mathrm{~s}^{-1}, J\left(\mathrm{gm} \mathrm{s}^{-1}\right)=-5.45$ dex, corresponding to $\Phi_{\mathrm{RL}}=$ $2.5 \times 10^{-6} M_{\odot} / \mathrm{yr}$. The initial condition for the integration of Eq. (2) is obtained from the RL at the point $P_{1}$ where $v=3 a$. Thus $v_{1}=52.5 \mathrm{~km} \mathrm{~s}^{-1}$ and this occurs at $x_{1}=0.950$.

\subsection{Initial derivative}

Because of the dependence of $g_{\ell}$ on $v^{\prime}$, specifying $P_{1}$ does not uniquely determine the trajectory $v(x ; \Phi)$ for $x<x_{1}$ - i.e., the wind's velocity law. This is illustrated in Fig. 1, which plots the function $Q_{1}(z)$ obtained from Eq. (7) by setting $(v, x, \Phi)=$ $\left(v_{1}, x_{1}, \Phi_{\mathrm{RL}}\right)$. We see that the equation $Q_{1}=0$ has two roots, $z_{\mathrm{R}}=1.81$ and $z_{\mathrm{S}}=-0.25$, corresponding to rapid and slow initial accelerations, respectively.

Figure 2 shows how $z_{\mathrm{R}}$ and $z_{\mathrm{S}}$ vary with $\Phi$. The roots coalesce in a double root at $z_{*}=0.78$ when $\Phi=\Phi_{*}=$ $1.05 \times 10^{-5} M_{\odot} /$ yr. For $\Phi>\Phi_{*}$, there is no root and so a matching supersonic solution does not then exist. On the other hand, for $\Phi<\Phi_{*}$, there are two solutions given by the $R$ - and $S$-branches. The appropriate continuation is the one already selected by the RL solution as it enters the Sobolev regime. For the S-30 model in Paper I, the velocity derivative at $v=3 a$ gives $z_{1}=1.42$ for $\Phi_{\mathrm{RL}}=-5.60 \mathrm{dex}$, and this point is plotted in Figs. 1 and 2. Clearly, the point on the $R$-branch is the appropriate continuation.

\subsection{Velocity law}

With the $R$-branch thus selected, Eq. (2) is integrated outwards from $P_{1}$ in order to determine $v\left(x ; \Phi_{\mathrm{RL}}\right)$. As at $P_{1}$, Eq. (7) continues to have two roots $z_{\mathrm{S}}$ and $z_{\mathrm{R}}$ until $v \simeq 140 \mathrm{~km} \mathrm{~s}^{-1}$, at which

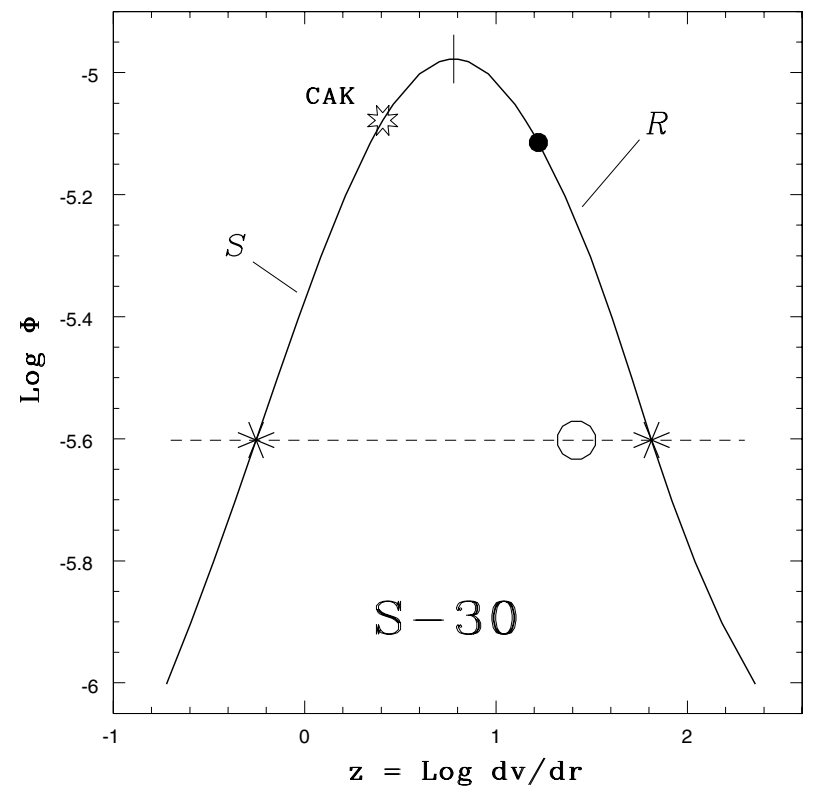

Fig. 2. Velocity gradients satisfying the equation $Q_{1}=0$ at $P_{1}$ as functions of $\Phi$. The $R$ - and $S$-branches are indicated. The asterisks and the open circle are for $\Phi=\Phi_{\mathrm{RL}}$, as in Fig. 1. The star on the $S$-branch is the CAK solution. Points on the $R$-branch below the filled circle give singularity-free winds extending to $r=\infty$.

point $z_{\mathrm{S}} \rightarrow-\infty$. The steeper of the two possible velocity gradients is selected as the natural continuation of the intial choice of $z_{\mathrm{R}}$. The integration is continued to $r \gtrsim 200 R$, giving a terminal velocity $v_{\infty}=1820 \mathrm{~km} \mathrm{~s}^{-1}$. If the velocity law is then fitted to the standard form $v=v_{\infty}(1-x)^{\beta}$ at $v / v_{\infty}=0.5$, we obtain $\beta=0.69$. These results are unexceptional.

But of exceptional interest is the integration's successful continuation to $r=\infty$, apparently without encountering a singularity. Because the root-finding uses Newton-Raphson (N-R) iterations, a convergence failure would occur at a singularity, thus terminating the integration at finite $r$.

\subsection{Locus of singular points}

In order to confirm that $v\left(x ; \Phi_{\mathrm{RL}}\right)$ does not cross a locus of singular points, the nearest such points are now found.

When the integration reaches the point $\xi_{i}=\left(v_{i}, x_{i}, \Phi_{\mathrm{RL}}\right)$, the equation $Q\left(z_{i}, \boldsymbol{\xi}_{\boldsymbol{i}}\right)=0$ is solved for $z_{i}$, thus determining $v^{\prime}$ for the next integration step. But now $z$ is varied away from $z_{i}$ in order to explore the solution topology along the line $\boldsymbol{\xi}=\xi_{i}$ in $\left(v^{\prime}, v, x, \Phi\right)$ space. Thus we define a sequence of functions $Q_{i}(z)=Q\left(z ; \boldsymbol{\xi}_{i}\right)$ of the single variable $z$, the first of which $Q_{1}(z)$ is already plotted in Fig. 1.

In Fig. 3, this $Q_{i}$-sequence is plotted for a dense set of points $P_{i}$ through the wind, and the stationary points $z_{i}^{s}$ of the $Q_{i}$ are marked. Since the curve defined by the $z_{i}^{s}$ does not intersect the line $Q=0$, these calculations show that nowhere along the supersonic wind is $\partial Q / \partial v^{\prime}=0$, thus confirming the absence of a singularity and explaining the success of N-R iterations in extracting $v^{\prime}$.

\subsection{Singularity-free solutions}

Sections 3.3 and 3.4 show that the moving-RL for S-30 can be matched to a singularity-free wind extending to $r=\infty$. 


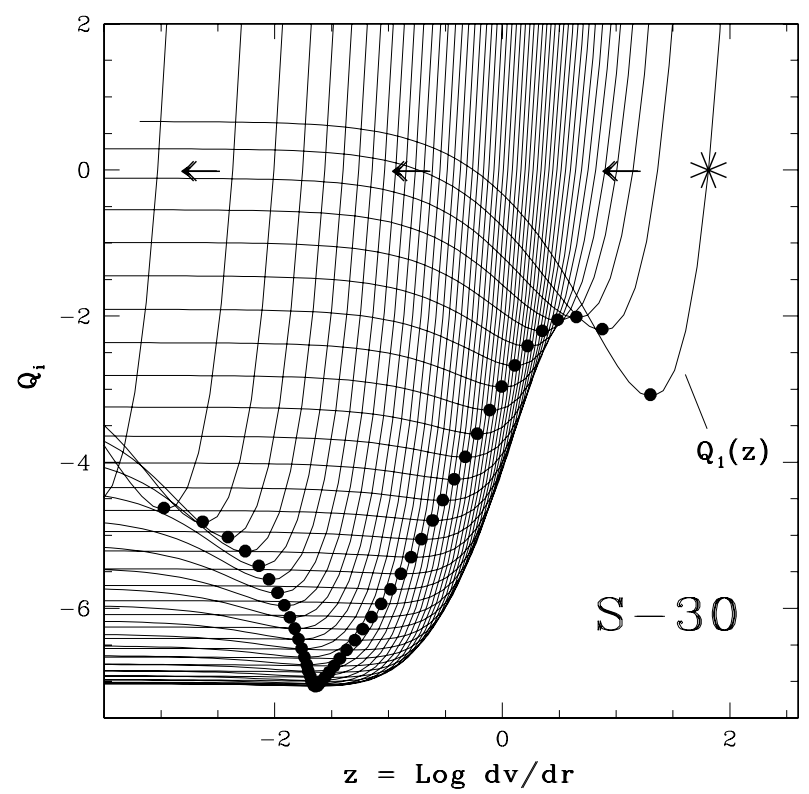

Fig. 3. Functions $Q_{i}(z)$ for points $\left(v_{i}, x_{i}\right)$ throughout the supersonic wind of model S-30. The asterisk is the initial gradient $z_{\mathrm{R}}$ and the corresponding $Q_{1}(z)$ is labelled - see also Fig. 1. The arrows indicate the direction of increasing $v$ along the line $Q=0$ and the filled circles locate the stationary points $z_{i}^{s}$ of the $Q_{i}(z)$.

To explore how special such a continuation is, a sequence of outward integrations from $P_{1}$ is carried out with $\Phi$ as parameter. For each integration, the starting derivative is the $R$-root from Fig. 2, and the integration continues with this root as in Sect. 3.3. From this sequence, the singularity-free domain is found to be $\Phi \leq \Phi_{u}=7.68 \times 10^{-6} M_{\odot} /$ yr. This upper limit is indicated on the $R$-branch in Fig. 2 . The corresponding terminal velocity is $\left(v_{\infty}\right)_{u}=887 \mathrm{~km} \mathrm{~s}^{-1}$.

An integration with $\Phi$ increased to $7.69 \times 10^{-6} M_{\odot} /$ yr encounters a singularity when $v=102.5 \mathrm{~km} \mathrm{~s}^{-1}$, at which point the $\mathrm{N}-\mathrm{R}$ iterations fail to converge. Interestingly, the proximity of a singularity is evident in the successful integration for $\Phi=\Phi_{u}$ in the form of a sharp decrease in $\mathrm{d} v / \mathrm{d} r$ at $v \sim 105 \mathrm{~km} \mathrm{~s}^{-1}$. Clearly, $\Phi_{u}$ is the value of $\Phi$ such that the outward integration just grazes the locus of singular points. The velocity of this tangent point for S-30 is $v_{u} \simeq 104 \mathrm{~km} \mathrm{~s}^{-1}$.

Because $\Phi_{u}$ comfortably exceeds $\Phi_{\mathrm{RL}}$, the successful continuation of the RL is not fortuitous. Such continuations can therefore be anticipated for RL's covering a wide range of $v_{t}$.

\subsection{Abbott waves}

With radiative transfer treated using the Sobolev approximation, Abbott (1980) studied the propagation of density fluctuations in line-driven winds. He demonstrated that spherically-symmetric radiative-acoustic waves are non- dispersive and propagate in the local matter frame with velocities $C_{+}$and $-C_{-}$, given by

$C_{-}=w+\sqrt{w^{2}+a^{2}}$ and $C_{+}=-w+\sqrt{w^{2}+a^{2}}$

where $w=1 / 2 \partial g_{\ell} / \partial v^{\prime}$. Transforming to the star's frame, we see that information can propagate inwards towards the star if $u<0$, where

$u=v-C_{-}$.

From Eqs. (8)-(10), we readily recover Abbott's major result that $u=0$ when $\partial Q / \partial v^{\prime}=0$. He thereby demonstated that the CAK critical point is the furthest point downstream that can communicate with and therefore influence every other point in the wind. This physical interpretation of what had hitherto only had a mathematical justification is undoubtedly a major reason for the almost universal acceptance of CAK theory as the correct theoretical foundation for line-driven winds and, in particular, of the CAK critical point's role in determining $\Phi$.

Abbott's analysis can be applied to study information flow in the singularity-free supersonic wind of S-30. Points in the wind where $u>0$ cannot communicate with the upstream flow. From Eqs. (8)-(10), we find that

$u>0$ if $\frac{\partial Q}{\partial v^{\prime}}>0$ when $Q=0$.

This propagation criterion can be applied to Fig. 3 . We see that the $R$-root intersections of the curves $Q_{i}(z)$ with the line $Q=0$ are all such that $\mathrm{d} Q_{i} / \mathrm{d} z>0$. Thus no point in this solution can influence the upstream structure by means of Abbott waves. In particular, no information propagates back into the RL across the boundary at $x_{1}$.

Figure 3 suffices for these qualitative remarks. The actual propagation speeds are as follows: $u=17.4 \mathrm{~km} \mathrm{~s}^{-1}$ at $P_{1}$, increases to a maximum of $705.2 \mathrm{~km} \mathrm{~s}^{-1}$ when $v=1644 \mathrm{~km} \mathrm{~s}^{-1}$, then decreases to $\sim 620 \mathrm{~km} \mathrm{~s}^{-1}$ in the terminal flow.

The propagation speeds are also of interest for the limiting singularity-free solution with $\Phi=\Phi_{u}$ (Sect. 3.5). Initially, $u=11.4 \mathrm{~km} \mathrm{~s}^{-1}$ at $P_{1}$, drops to a minimum of $0.8 \mathrm{~km} \mathrm{~s}^{-1}$ at $v=104.5 \mathrm{~km} \mathrm{~s}^{-1}$, then rises monotonically to $217 \mathrm{~km} \mathrm{~s}^{-1}$ in the terminal flow. This near approach to $u=0$ reflects this solution's close proximity to the singular locus when $v \sim 105 \mathrm{~km} \mathrm{~s}^{-1}$.

The propagation criterion can also be applied to Figs. 1 and 2 . The $S$-roots of the equations $Q(z ; \Phi)=0$ are such that $\mathrm{d} Q / \mathrm{d} z<0$, so $u<0$ and information can propagate back into the RL. The opposite holds for the $R$-roots. When the roots coalesce, the double root is such that $\mathrm{d} Q / \mathrm{d} z=0$, whence $u=0$ and $P_{1}$ is a singularity.

\subsection{CAK solution}

As noted above, Abbott demonstrated that $u$ changes sign at $v_{\text {CAK }}$, the velocity of the CAK critical point, from being $<0$ when $v<v_{\text {CAK }}-$ i.e., in the upstream sub-critical flow, to being $>0$ when $v>v_{\text {CAK }}-$ i.e., in the downstream super-critical flow. Thus, the CAK solution constrained to pass through $P_{1}$ must correspond to a point on the $S$-branch of Fig. 2. Accordingly, shooting integrations starting at $P_{1}$ with the $S$-root $z_{\mathrm{S}}(\Phi)$ are used as shown in Fig. 4 to bracket the value of $\left(\Phi_{\mathrm{CAK}}\right)_{1}$. To sufficient accuracy, $\left(\Phi_{\mathrm{CAK}}\right)_{1}=8.35 \times 10^{-6} M_{\odot} / \mathrm{yr}$, and this is plotted on the $S$-branch in Fig. 2.

This calculation shows that a CAK-continuation of the moving-RL fails on two counts. Not only is $\left(\Phi_{\mathrm{CAK}}\right)_{1} \neq \Phi_{\mathrm{RL}}$ as anticipated in Sect. 1, but the respective solution branches also differ: the sub-critical zones of CAK winds are on the $S$-branch, whereas the RL enters the Sobolev regime on the $R$-branch.

\section{Additional models}

The investigation of S-30 in Sect. 3 provides strong support for the approach in Paper I - i.e, determining $J$ as an eigenvalue of the equations governing the structure of the moving-RL, assuming no influence from the highly supersonic exterior flow. First, a singularity-free supersonic solution extending to $r=\infty$ exists with $\Phi=\Phi_{\mathrm{RL}}$. Second, this solution is everywhere super-critical 


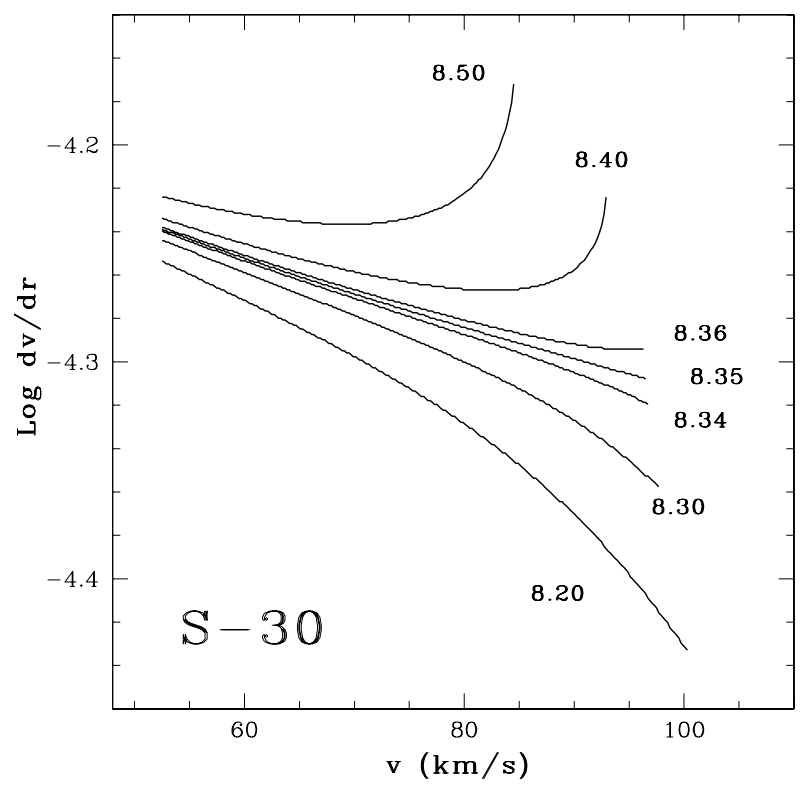

Fig. 4. Shooting integrations to determine $\left(\Phi_{\mathrm{CAK}}\right)_{1}$, all starting at $P_{1}$. The curves are labelled with $\Phi$ in units of $10^{-6} M_{\odot} / \mathrm{yr}$.

in regard to Abbott waves, which therefore carry no information back into the RL.

In order to demonstrate that this support is not restricted to S-30, identical calculations are reported for the two other Pauldrach et al. models considered in Paper I, namely D-40 and D-50. Numerical details for all three models are given in Table 1. Velocities are in $\mathrm{km} \mathrm{s}^{-1}$, mass fluxes in $\mathrm{gm} \mathrm{s}^{-1}$ and mass-loss rates in $M_{\odot} / \mathrm{yr}$.

\subsection{Comments}

Some noteworthy aspects of Table 1 are as follows:

a) The high $v_{\infty}$ for D-40 suggests that $\Phi_{R L}$ is too low, but may also be a consequence of the no attenuation assumption (Sect. 2.2) retained from CAK.

b) For all three models, $\Phi_{\mathrm{RL}}<\Phi_{u}$ so that matching singularityfree exterior solutions exist. Moreover, we can anticipate that this remains true for the reduced $\Phi_{\mathrm{RL}}$ 's corresponding to higher $v_{\mathrm{t}}$ 's (see Table 1, Paper I).

c) Comparing the values of $z_{1}$ with the roots $z_{\mathrm{S}}$ and $z_{\mathrm{R}}$, we see that in each case the RL selects the $R$-root as the appropriate continuation to highly supersonic velocities. The existence of two barriers to matching onto a CAK exterior solution is therefore further supported.

d) In a matched solution, the last point able to communicate with every other point in the wind is the sonic point, where $v$ is still only a few percent of $v_{\text {esc }}$. This contrasts dramatically with CAK's O5f model (similar to D-50) where the corresponding last point $r_{\mathrm{CAK}}$ is at $v_{\mathrm{CAK}} \simeq 950 \mathrm{~km} \mathrm{~s}^{-1}$, a velocity well in excess of $v_{\text {esc }}\left(r_{\mathrm{CAK}}\right)$. Accordingly, no matter moving with $v<v_{\text {esc }}(r)$ is out of contact with the upstream flow, suggesting perhaps a mechanism that adjusts the wind's base structure to ensure eventual escape. But this possibly appealing aspect of the original CAK theory does not survive subsequent modifications, especially those of Pauldrach et al. (1986) - see their Table 1 and Fig. 2a.

e) Because escape is not guaranteed for matter at sonic velocity in the RL's, the existence of a matching supersonic
Table 1. O-star winds.

\begin{tabular}{cccc}
\hline \hline Quantity & S-30 & D-40 & D-50 \\
\hline \hline$T_{\text {eff }}$ & 30000 & 40000 & 50000 \\
$\log g$ & 3.00 & 3.75 & 4.00 \\
$R / R_{\odot}$ & 27 & 10 & 12 \\
$v_{\text {esc }}$ & 423 & 743 & 998 \\
$a$ & 17.5 & 20.2 & 22.6 \\
$v_{\mathrm{t}}$ & 10 & 10 & 10 \\
$\log J$ & -5.45 & -5.65 & -4.41 \\
& & & \\
$x_{1}$ & 0.950 & 0.978 & 0.990 \\
$v_{1}$ & 52.5 & 60.6 & 67.7 \\
$z_{\mathrm{S}}$ & -0.25 & -0.19 & 0.39 \\
$z_{\mathrm{R}}$ & 1.81 & 2.64 & 1.82 \\
$z_{1}$ & 1.42 & 1.97 & 1.84 \\
$\Phi_{\mathrm{RL}}$ & $2.5 \times 10^{-6}$ & $0.22 \times 10^{-6}$ & $5.4 \times 10^{-6}$ \\
$v_{\infty}$ & 1820 & 5094 & 2578 \\
$\beta$ & 0.69 & 0.61 & 0.63 \\
$z_{*}$ & 0.78 & 0.90 & 1.06 \\
$\Phi_{*}$ & $1.05 \times 10^{-5}$ & $2.71 \times 10^{-6}$ & $1.06 \times 10^{-5}$ \\
$\Phi_{u}$ & $7.68 \times 10^{-6}$ & $0.86 \times 10^{-6}$ & $7.69 \times 10^{-6}$ \\
$\left(v_{\infty}\right)_{u}$ & 887 & 2442 & 2180 \\
$v_{u}$ & $\simeq 104$ & $\simeq 168$ & $\simeq 189$ \\
$\left(\Phi_{\mathrm{CAK}}\right)_{1}$ & $8.35 \times 10^{-6}$ & $1.16 \times 10^{-6}$ & $8.1 \times 10^{-6}$ \\
$\left(v_{\mathrm{CAK}}\right)_{1}$ & $\simeq 97$ & $\simeq 159$ & $\simeq 156$ \\
& & & \\
\hline & & &
\end{tabular}

solution is essential in justifying the assumption of stationarity. If there is no such exterior solution, we should expect intermittency due to matter falling back onto the photosphere as well as departures from spherical symmetry due to a loss of phase coherence over the stellar surface.

Moving RL's with no matching supersonic wind will probably be found. However, for the three models in Table 1, exterior solutions do exist. The reasons are well understood: with only modest changes in $W / n_{\mathrm{e}}$, the ratio of dilution factor to electron density, the ionization balance throughout these winds is such that matter remains an efficient scatterer of UV radiation, and Doppler shifts continually irradiate lines with fresh unattenuated continuum.

\section{Conclusion}

The aim of this paper has been to investigate the problem of matching the moving-RL's of Paper I to supersonic winds extending to $r=\infty$. Perhaps surprisingly, matching exterior solutions have been obtained with a modified CAK procedure using the Sobolev approximation. But in contrast to standard CAK theory, the entire solution thus constructed has the same criticalpoint structure as the basic integro-differential system. Thus the sonic point retains its role as a critical point (Paper I), and a CAK critical point no longer appears in the supersonic flow (Sect. 3.5). In consequence, there is a reasonable expectation that such solutions will be good approximations to solutions obtained with non-Sobolev transfer (Sect. 2.3).

Use of a modified CAK procedure for the exterior solutions has allowed comparisons with CAK solution topology and, crucially, with Abbott's discussion of information propagation by 
radiative-acoustic waves. With these issues now clarified, future calculations should re-introduce multi-line scattering. This can be done by extending the Monte Carlo procedures of Paper I to $r=\infty-$ see also Abbott \& Lucy (1985) and Vink et al. (2000). Another attractive possibility is to revive the comoving frame approach (Mihalas et al. 1975; Weber 1981; Pauldrach et al. 1986). In either case, $\Phi$ is determined by the constraint of regularity at the sonic point and not at the CAK critical point, which no longer exists. Accordingly, scaling relations for $\Phi$ need to be re-investigated since the basis is undermined for those obtained from the CAK critical point.

Acknowledgements. The referee, S.P. Owocki, is thanked for his comments and suggestions. This investigation also benefited from points raised by J. Puls in his report on Paper I.

\section{References}

Abbott, D. C. 1980, ApJ, 242, 1183

Abbott, D. C., \& Lucy, L. B. 1985, ApJ, 288, 679

Castor, J. I., Abbott, D. C., \& Klein, R. I. 1975, ApJ, 195, 157 (CAK)

Kurucz, R. L., \& Bell, B. 1995, Kurucz CD-ROM No.23

Lanz, T. \& Hubeny, I. 2003, ApJS, 146, 417

Lucy, L. B. 1971, ApJ, 163, 95

Lucy, L. B. 1975, Mem. Soc. R. Sci. Liège, 8,3 59

Lucy, L. B. 1999, A\&A, 345, 211

Lucy, L. B. 2007, A\&A, 468, 649 (Paper I)

Lucy, L. B. \& Solomon, P. M. 1970, ApJ, 159, 879

Mihalas, D., Kunasz, P. B., \& Hummer, D. G. 1975, ApJ, 202, 465

Pauldrach, A. W. A., Hoffmann, T. L., \& Lennon, M. 2001, A\&A, 375, 161

Pauldrach, A., Puls, J., \& Kudritzki, R. P. 1986, A\&A, 164, 86

Weber, S. V. 1981, ApJ, 243, 954

Vink, J. S., de Koter, A., \& Lamers, H. J. G. L. M. 2000, A\&A, 362, 295 